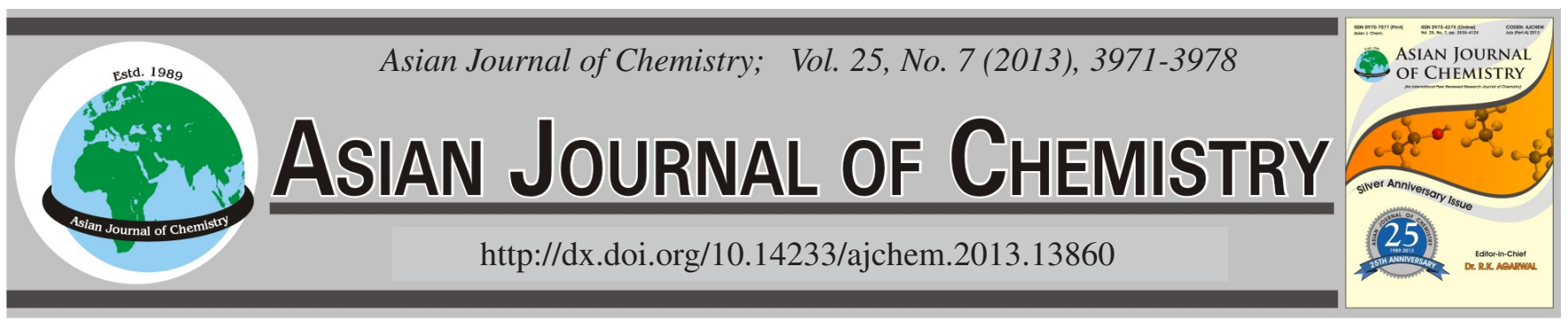

\title{
Antioxidant Capacity of Caffeic Acid, Phloretin and Glutathione Mixtures and Formula Optimization
}

\begin{abstract}
YUE SUN ${ }^{1}, Y_{A N-Y I N G ~ Y U^{1, *} \text { and SHU-Wen CAO }}{ }^{1,2}$
${ }^{1}$ Department of Chemistry, Nanchang University, Nanchang 330031, P.R. China

${ }^{2}$ State Key Laboratory of Food Science and Technology, Nanchang University, Nanchang 330047, P.R. China

*Corresponding author: Fax: +86 791 88170312; Tel: +86 791 83969610; E-mail: yuyanying@ncu.edu.cn; sunyue_2009@163.com

In this paper, the synergistic antioxidant effect of caffeic acid, phloretion and glutathione was studied. Three different models, 2,2diphenyl-1-picrylhydrazyl (DPPH), reducing power and Trolox equivalent antioxidant capacity, were used to evaluate the antioxidant capacity of the antioxidants and their combinations. According to the results, not only formula with different proportions of antioxidants showed different synergistic effects in the same model, but also the same formula showed different synergistic effects in different models. By considering the antioxidation mechanism, it found that the antioxidant synergism effect which could enhance the antioxidant effectiveness of natural antioxidants was attributed to the regeneration of the different antioxidants. Principal component analysis combined with response surface methodology (PCA-RSM), overall desirability combined with response surface methodology (OD-RSM) and full probability evaluation combined with response surface methodology (FPE-RSM) methods were used to optimize formula, respectively. From the results of FPE-RSM method, the optimal formula contains caffeic acid $47.8 \%$, phloretin $29.6 \%$ and glutathione $22.6 \%$. Our research will help to select the optimal formula and make advanced antioxidant additives cosmetic system.

Key Words: Antioxidants, Synergistic effects, Caffeic acid, Phloretin, Glutathione.
\end{abstract}

\section{INTRODUCTION}

In recent years, the antioxidants have attracted wide attention for their important effect on drugs and cosmetic. First, they can prevent some targeted drugs or active component in cosmetics (such as amino acids, no saturated paraffin, aromatic hydrocarbons and active protein, etc.) from deterioration which is caused by bacterial invasion in the air or photocatalytic degradation. Second, they work together with other active substrates in health medicine to moisturizing, facial lifting, antiaging and inhibit cancerous and atherosclerosis. Due to the potentially toxic and carcinogenic effects of the synthetic antioxidants such as BHA, BHT and TBHQ, natural antioxidants are receiving more and more attention. Natural antioxidants may be replacing the synthetic antioxidants. The researches have paid more attention to the "multi-antioxidants hypothesis". According to this hypothesis, better effect can be achieved when multiple antioxidants are used simultaneously. In this state, they can significantly improve the activity of each other and maintain excellent antioxidant equilibrium state of body ${ }^{1}$. According to the previous literatures, the effect of combination of several antioxidants is better than that of single antioxidant ${ }^{2}$. It has received extensive attention in the food industry. Zhou et al. $^{3}$ studied the antioxidant activity of polyphenols (contain catechin, epicatechin, catechin gallate, epicatechin gallate and gallic acid), L-ascorbic acid and vitamin $\mathrm{E}$ in linoleic acid sodium dodecyl sulphate (SDS) micelles. In this system, polyphenols can regenerate vitamin $\mathrm{E}$ and L-ascorbic acid can reduce polyphenols oxidized, which form antioxidant cycle. Romano, et $a l .{ }^{4}$ found that rosemary extract combine with BHT (butylated hydroxytoluene) and BHA (butylated hydroxyanisole) can achieve good synergism effect. Becker et al..$^{5}$ studied the antioxidant synergism effect of quercetin, $\alpha$-tocopherol and rutin in lipid systems. They found that the antioxidant synergism effect was operated by improving the sorting of lipid substrate. Schroeder et al. ${ }^{6}$, found that fertility trihydroxybenzene and carotenoids in palm oil have a good synergistic antioxidant effect. All above findings were limited to two components. Rare reports were about the interaction of three or more antioxidant components except by Liu et al. ${ }^{7}$.

When the body is exposure to ultraviolet irradiation, it can produce superoxide free radicals $\left(\mathrm{O}_{2}{ }^{-}\right)$, hydroxyl $\left(\mathrm{HO}^{\circ}\right)$, peroxyl free radicals $\left(\mathrm{HOO}^{\circ}\right)$, alkoxyl free radicals ( $\left.\mathrm{RO}^{\circ}\right)$, alkyl free radicals $\left(\mathrm{R}^{\circ}\right)$, sulphur and carbon free radicals ${ }^{8}$. These free radicals can lead to premature aging and skin cancer. The 
skin possesses intrinsic antioxidant systems to protect itself from oxygen species (ROS) produced by UV induced. However, the capacity of these systems is restrained when the skin is exposure to irradiation for long time. The endogenous defence systems included various low molecular weight antioxidants (ascorbate, glutathione, $\alpha$-tocopherol, ubiquinol and $\beta$-carotene) and antioxidant enzymes (superoxide dismutase, catalase, glutathione peroxidase, glutathione reductase and thioredoxin reductase). After excessive exposure to UV irradiation, these skin antioxidants are depleted ${ }^{9}$ and cause free radicals to hurt skin. Exogenous antioxidants system assists with endogenous defense systems protected our skin from damage caused by free radicals. Glutathione (GSH) is a new cutaneous antioxidant ${ }^{10}$, which can antiaging, antioxidation ${ }^{11}$. It can be used in cosmetics to enhance skin elasticity, shrink pore. Recently researches illustrate that it has effect on asthma ${ }^{12}$ and maintenance of the mitochondrial genome ${ }^{13}$. Caffeic acid (CAF) and phloretin (PHL) are exogenous antioxidants. Phloretin is a new type of natural food antioxidant factors, which is found in juicy fruit peel and root skin. It has been attracted wide attention because of its function in antioxidant ${ }^{14}$, antimutagenic $^{15}$, antiinflammatory and immunosuppressive effect ${ }^{16}$. It can be used to moisturiz and promote the absorption ability of other functional factors in the formula. It inhibits excessively secrete of skin glands throuth prevent carbohydrate components into the epidermal cells. Caffeic acid is a common additive in whiten can be found in many traditional Chinese medicine. Its main function is to scavenge reactive oxygen species (ROS) $)^{17}$. Moreover, it often be used to antibacterial and inhibit activity of tyrosine kinase against ischemiareperfusion injury ${ }^{18,19}$.

Although a combination of the three substrates is common in cosmetics system, it has no report about the synergistic antioxidant effect of them. The purpose of this study is to explore the existence of antioxidant synergistic effects based on different combinations, using the vivo antioxidant capacity assay and try to formula optimization. That can provide the basis for development of cosmetics formula based on the synergistic effect. Molecular structures of three antioxidants are shown in Fig. 1.<smiles>O=C(CCc1ccc(O)cc1)c1c(O)cc(O)cc1O</smiles>

Phloretin<smiles>O=C(O)/C=C/c1ccc(O)c(O)c1</smiles>

Caffeic acid<smiles>N[C@@H](CCC(=O)N[C@@H](CS)C(=O)NCC(=O)O)C(=O)O</smiles>

Glutathione

Fig. 1. Chemical structures of caffeic acid, phloretin and glutathione

\section{EXPERIMENTAL}

Caffeic acid (CAF, > $98 \%$ ) and phloretin (PHL, > $98 \%$ ) were purchased from Shaanxi HuaTai Bio-fine chemical company Ltd. (Shaanxi, China). Glutathione (GSH, > 98 \%) was purchased from Shaanxi Sciphar Biotechnology Co., Ltd. (Shaanxi, China). DPPH, Trolox and ABTS were purchased from Sigma Chemical Co. (St. Louis, MO, USA). Ethanol (90\%), sodium dihydrogen phosphate, disodium hydrogen phosphate and ferric chloride were purchased from Xi-long Chemical Factory (Guangdong, China). Potassium persulphate and potassium ferrocyanide were obtained from Guangzhou Chemical Reagent (Guangzhou, China). Trichloroacetic acid was bought from Tianjin Da-mao Chemical Reagent Factory (Tianjin, China). Water was purified using a Milli-Q system (Millipore, Molsheim, France).

Antioxidant solution: 30 formulas were designed based on the alteration of combination ratio of mixed antioxidants and the composition was shown in Table-1. Stock solutions of caffeic acid $(10 \mu \mathrm{mol} / \mathrm{mL})$, glutathione $(10 \mu \mathrm{mol} / \mathrm{mL})$ and phloretin $(10 \mu \mathrm{mol} / \mathrm{mL})$ were dissolved in ethanol/water $(40 \%$, v/v) and stored at $4{ }^{\circ} \mathrm{C}$. The required amount of antioxidant mixtures were prepared by mixing the appropriate amount of each stock solution. All stock solutions were stirred at room temperature for $10 \mathrm{~min}$ before further use.

DPPH assay: DPPH (1,1-diphenyl-2-picryl-hydrazyl) was characterized as a stable free radical by virtue of the delocalization of the spare electron over the molecule as a whole, so that the molecules don't dimerize, as would be the case with most other free radicals. When a solution of DPPH was mixed with that of a substance that can donate a hydrogen atom, then this gives rise to the reduced form with the loss of this violet colour and there would be expected to be a residual pale yellow colour ${ }^{20}$. The radical-scavenging capacity was determined according to the procedure of Maisuthisakul et al. ${ }^{21}$ with slight modifications. DPPH radical in ethanol $\left(6 \times 10^{-5}\right.$ $\mathrm{mol} / \mathrm{L})$ was prepared and this solution $(0.5 \mathrm{~mL})$ was added to sample solutions in ethanol, then the total volume was $5 \mathrm{~mL}$. These antioxidant mixtures were subjected to preincubation at room temperature for $0.5 \mathrm{~h}$ in the dark, absorbance was measured at $517 \mathrm{~nm}$ with a UV-2450 spectrophotometry (Shimadzu, Japan). DPPH radical scavenging activity was measured in triplicate sample. The percentage of DPPH radical scavenging activity was calculated using the following equation:

DPPH radical scavenging activity $(\%)$

$$
=\left[\frac{\mathrm{Abs}_{(\text {control })}-\mathrm{Abs}_{(\text {standard })}}{\mathrm{Abs}_{(\text {control })}}\right] \times 100
$$




\begin{tabular}{cccccccccccccccc}
\hline & \multicolumn{10}{c}{ TABLE-1 } \\
\multicolumn{110}{c}{ Code } & A1 & A2 & A3 & A4 & A5 & A6 & A7 & A8 & A9 & A10 & A11 & A12 & A13 & A14 & A15 \\
\hline $\mathrm{PHL}\left(\mathrm{X}_{1}\right)(\mathrm{C} / \mathrm{C} \%)$ & 100 & 0 & 0 & 50 & 50 & 0 & 33.3 & 66.7 & 16.7 & 16.7 & 33.3 & 66.7 & 33.3 & 66.7 & 0 \\
$\mathrm{GSH}\left(\mathrm{X}_{2}\right)(\mathrm{C} / \mathrm{C} \%)$ & 0 & 100 & 0 & 50 & 0 & 50 & 33.3 & 16.7 & 66.7 & 16.7 & 66.7 & 33.3 & 0 & 0 & 33.3 \\
$\mathrm{CAF}\left(\mathrm{X}_{3}\right)(\mathrm{C} / \mathrm{C} \%)$ & 0 & 0 & 100 & 0 & 50 & 50 & 33.3 & 16.7 & 16.7 & 66.7 & 0 & 0 & 66.7 & 33.3 & 66.7 \\
\hline $\mathrm{Code}$ & $\mathrm{A} 16$ & $\mathrm{~A} 17$ & $\mathrm{~A} 18$ & $\mathrm{~A} 19$ & $\mathrm{~A} 20$ & $\mathrm{~A} 21$ & $\mathrm{~A} 22$ & $\mathrm{~A} 23$ & $\mathrm{~A} 24$ & $\mathrm{~A} 25$ & $\mathrm{~A} 26$ & $\mathrm{~A} 27$ & $\mathrm{~A} 28$ & $\mathrm{~A} 29$ & $\mathrm{~A} 30$ \\
\hline $\mathrm{PHL}\left(\mathrm{X}_{1}\right)(\mathrm{C} / \mathrm{C} \%)$ & 0 & 16.7 & 50 & 16.7 & 40 & 40 & 60 & 0 & 0 & 83.3 & 16.7 & 40 & 30 & 30 & 20 \\
$\mathrm{GSH}\left(\mathrm{X}_{2}\right)(\mathrm{C} / \mathrm{C} \%)$ & 66.7 & 33.33 & 33.33 & 50 & 20 & 60 & 0 & 60 & 16.7 & 16.7 & 0 & 30 & 40 & 30 & 30 \\
$\mathrm{CAF}\left(\mathrm{X}_{3}\right)(\mathrm{C} / \mathrm{C} \%)$ & 33.3 & 50 & 16.7 & 33.33 & 40 & 0 & 40 & 40 & 83.3 & 0 & 83.3 & 30 & 30 & 40 & 50 \\
\hline The proportion of each component in composition based on concentration $(\mathrm{C} / \mathrm{C} \%)$. Final concentration $(\mu \mathrm{mol} / \mathrm{L})$ of each composition in three
\end{tabular}

antioxidant model was DPPH model: $3.6 \mu \mathrm{mol} / \mathrm{L}$; reducing power model: $37.5 \mu \mathrm{mol} / \mathrm{L}$; TEAC model: $1.8 \mu \mathrm{mol} / \mathrm{L}$.

where $\mathrm{Abs}_{\text {(control) }}$ was the absorbance of the control solution containing DPPH radical, $\mathrm{Abs}_{\text {(standard) }}$ was the absorbance of the mixtures containing DPPH radical and antioxidant after $0.5 \mathrm{~h}$ without blank.

Reducing power assay: The reducing power of the sample was determined according to the follow method ${ }^{22}$. Different concentrations of the sample in $2.5 \mathrm{~mL}$ of solvent were mixed with phosphate buffer $(2.5 \mathrm{~mL}, 0.2 \mathrm{M}, \mathrm{pH}$ 6.6) and potassium ferrocyanide $(2.5 \mathrm{~mL}, 1 \%)$. The mixture was incubated at $50{ }^{\circ} \mathrm{C}$ for $20 \mathrm{~min}$. A portion of trichloroacetic acid $(2.5 \mathrm{~mL}, 10 \%)$ was added to the mixture, which was then centrifuged at $3000 \mathrm{rpm}$ for $10 \mathrm{~min}$. The upper layer of the solution $(2.5 \mathrm{~mL})$ was mixed with distilled water $(2.5 \mathrm{~mL})$ and $\mathrm{FeCl}_{3}(0.5 \mathrm{~mL}, 0.1 \%)$ and the absorbance was measured at $700 \mathrm{~nm}$ and compared with standards. Increased absorbance of the reaction mixture indicated increased reducing power.

Trolox equivalent antioxidant capacity assay: The Trolox equivalent antioxidant capacity (TEAC) assay was a rapid, easy and correlated with the biological activity of antioxidants method for assessing the capacity of a compound to scavenge ABTS radicals $\left(\mathrm{ABTS}^{\bullet+}\right)^{23}$. Detection method was as follows: $200 \mu \mathrm{L}$ samples was added in $10 \mathrm{~mL}$ cuvette and $4.5 \mathrm{~mL}$ $\mathrm{ABTS}^{\bullet+}$ radical solution were added immediately, then ethanol was added quickly to ensure the final volume was $5 \mathrm{~mL}$, at which point the antioxidants began to inhibit the radical, producing a reduction in absorbance. The reaction reacted under dim light and at room temperature and record absorbance after $6 \mathrm{~min}^{24}$. A quantitative relationship between the reduction and the concentration of antioxidants present in the sample. Inhibition was calculated using the following equation:

$$
\text { Inhibition }(\%)=\left[\frac{A_{0}-A_{1}}{A_{0}}\right] \times 100
$$

where $A_{0}$ was the absorbance of the control solution, $A_{1}$ was the absorbance of ABTS radical + sample/standard after 6 min without blank. At the same time, a Trolox calibration curve was prepared and the inhibition percentage obtained for the sample was interpolated to calculate the concentration in Trolox equivalents (TE). Each sample was analyzed in triplicate.

Preparation of the ABTS solution: A $7 \mathrm{mM}$ solution of ABTS in milli-Q was prepared and $\mathrm{ABTS}^{\bullet+}$ was formed after addition of potassium persulphate to the mixture in a final concentration of $2.45 \mathrm{mM}$. After $12-16 \mathrm{~h}$ incubation at room temperature, the stock solution was diluted with PBS until an absorbance of $0.7 \pm 0.02$ at $734 \mathrm{~nm}$ was reached ${ }^{25}$.

Calculation of synergistic effect of antioxidant mixtures: A Trolox calibration curve was prepared in each model and the inhibition percentage obtained for the sample was interpolated to calculate the concentration in Trolox equivalents (TE). According to above method, the experiment antioxidant capacity (EAC) of sample was obtained and all results were expressed in Trolox equivalents (TE, $\mu \mathrm{M})$. Each sample was analyzed in triplicate. The theoretical antioxidant capacity (TAC) is relate to the individual antioxidant, calculated using the following equation:

$$
\mathrm{TAC}=\mathrm{W}_{\mathrm{CAF}} \times \mathrm{T}_{\mathrm{CAF}}+\mathrm{W}_{\mathrm{PHL}}+\mathrm{T}_{\mathrm{PHL}}+\mathrm{W}_{\mathrm{GSH}} \times \mathrm{T}_{\mathrm{GSH}}
$$

where $\mathrm{T}_{\mathrm{CAF}}, \mathrm{T}_{\mathrm{PHL}}, \mathrm{T}_{\mathrm{GSH}}$ are representatives of antioxidant capacities generated a given dose of single caffeic acid, phloretin and glutathione, respectively. $\mathrm{W}_{\mathrm{CAF}}, \mathrm{W}_{\mathrm{PHL}}, \mathrm{W}_{\mathrm{GSH}}$ represent weight of caffeic acid, phloretin and glutathione in the formula (\%). The weight was based on the ratios of components in the formula.

The synergistic effect (SE) of a combination of antioxidants was based on the ratios of the experimental antioxidant capacity (EAC) and theoretical antioxidant capacity (TAC) and was calculated using the following equation ${ }^{26}$ :

$$
\mathrm{SE}=\frac{\mathrm{EAC}}{\mathrm{TAC}}
$$

where synergism was shown when $\mathrm{SE}$ was greater than 1, antagonism was shown when $\mathrm{SE}<1$.

Statistical analysis: All the experiments were performed at least in triplicate. Student's $t$-test was used for comparison between two means (EAC and TAC), using SPSS 16.0. A difference was considered statistically significant at $p<0.05$.

\section{RESULTS AND DISCUSSION}

DPPH model: To examine the SE of formula, the antioxidant capacities of all single antioxidants and mixtures were studied. Based on the data obtained from the individual antioxidants, the TAC was calculated at reaction end. If the EAC is the same as the theoretical values, then the contribution of the individual antioxidant would be additive. If the EAC is greater than the TAC value, then an interaction happened among the antioxidants, thus displaying synergism. Mathematically speaking, when a ratio of EAC/TAC $>1$, it would indicate an synergistic effect. The antioxidant formulae A7A10, A17-A20 and A27-A30 contained three antioxidants. In DPPH model, there were three mixtures (A7, A9 and A28) with ratios greater than 1 . A significant $(p<0.05) \mathrm{SE}$ was produced by the antioxidant formula $\mathrm{A} 9(\mathrm{SE}=1.11$; Table-2). Its $\mathrm{EAC}$ and TAC values were $4.45 \pm 0.10$ and $4.93 \pm 0.33$ $\mu \mathrm{M}$, respectively [Fig. 2(A)]. A7, A28 were not found to be 
TABLE-2

SYNERGISTIC EFFECT (SE) OF DIFFERENT COMPOSITIONS

\begin{tabular}{|c|c|c|c|c|c|c|}
\hline \multirow{2}{*}{ Code } & \multicolumn{2}{|c|}{ DPPH } & \multicolumn{2}{|c|}{ Reducing power } & \multicolumn{2}{|c|}{ TEAC } \\
\hline & $\mathrm{EAC}^{\mathrm{a}}(\mu \mathrm{mol})$ & $\mathrm{SE}^{\mathrm{b}}$ & $\mathrm{EAC}^{\mathrm{a}}(\mu \mathrm{mol})$ & $\mathrm{SE}^{\mathrm{b}}$ & $\mathrm{EAC}^{\mathrm{a}}(\mu \mathrm{mol})$ & $\mathrm{SE}^{\mathrm{b}}$ \\
\hline A1 & $2.54 \pm 0.18$ & - & $4.94 \pm 0.66$ & - & $9.36 \pm 0.21$ & - \\
\hline A2 & $2.96 \pm 0.11$ & - & $0.64 \pm 0.59$ & - & $4.88 \pm 0.48$ & - \\
\hline A3 & $12.31 \pm 0.70$ & - & $19.92 \pm 0.45$ & - & $1.25 \pm 0.37$ & - \\
\hline A4 & $2.48 \pm 0.03$ & $0.90 * *$ & $3.90 \pm 0.79$ & $1.40 *$ & $7.87 \pm 0.40$ & $1.11 *$ \\
\hline A5 & $6.99 \pm 0.30$ & 0.94 & $11.85 \pm 0.96$ & 0.95 & $6.39 \pm 0.09$ & $1.20 * * *$ \\
\hline A6 & $8.49 \pm 0.46$ & $1.11^{*}$ & $17.36 \pm 1.21$ & $1.69 * * *$ & $2.16 \pm 0.30$ & $0.70 * *$ \\
\hline A7 & $6.33 \pm 0.75$ & 1.07 & $14.47 \pm 1.08$ & $1.70 * * *$ & $5.83 \pm 0.54$ & 1.13 \\
\hline A8 & $3.79 \pm 0.24$ & $0.89 *$ & $10.10 \pm 0.96$ & $1.50 * *$ & $8.21 \pm 0.43$ & $1.13 *$ \\
\hline A9 & $4.93 \pm 0.33$ & $1.11 *$ & $11.92 \pm 0.62$ & $2.61 * * *$ & $5.09 \pm 0.29$ & 1.01 \\
\hline A10 & $7.82 \pm 0.58$ & $0.86^{*}$ & $17.01 \pm 0.50$ & $1.20 * * *$ & $4.61 \pm 0.51$ & $1.44 * *$ \\
\hline A11 & $1.89 \pm 0.06$ & $0.67 * * *$ & $5.23 \pm 1.11$ & $2.53 * *$ & $7.14 \pm 0.27$ & $1.12 *$ \\
\hline A12 & $2.07 \pm 0.15$ & $0.77 * *$ & $3.11 \pm 1.07$ & 0.89 & $8.24 \pm 0.57$ & 1.05 \\
\hline A13 & $6.42 \pm 0.52$ & $0.71 * *$ & $10.29 \pm 0.86$ & $0.69 * * *$ & $5.29 \pm 0.53$ & $1.34 * *$ \\
\hline A14 & $3.93 \pm 0.19$ & $0.68 * * *$ & $6.79 \pm 1.26$ & $0.68 * *$ & $8.18 \pm 0.56$ & $1.23 * *$ \\
\hline A16 & $3.73 \pm 0.25$ & $0.61 * * *$ & $19.01 \pm 1.13$ & $2.69 * * *$ & $2.39 \pm 0.47$ & $0.65 * *$ \\
\hline A17 & $5.70 \pm 0.16$ & $0.75 * * *$ & $17.06 \pm 2.00$ & $1.55^{* *}$ & $4.11 \pm 0.33$ & 1.08 \\
\hline A18 & $2.41 \pm 0.07$ & $0.56^{* * * *}$ & $14.34 \pm 1.20$ & $2.39 * * *$ & $7.03 \pm 0.55$ & 1.08 \\
\hline A19 & $3.69 \pm 0.16$ & $0.61 * * *$ & $18.99 \pm 0.46$ & $2.44 * * *$ & $4.66 \pm 0.21$ & 1.05 \\
\hline A20 & $3.94 \pm 0.54$ & $0.60 * *$ & $16.10 \pm 1.09$ & $1.60 * * *$ & $6.11 \pm 0.22$ & $1.17 * *$ \\
\hline A21 & $2.22 \pm 0.13$ & $0.80 * *$ & $3.38 \pm 1.75$ & $1.43 *$ & $7.64 \pm 0.20$ & $1.15^{* *}$ \\
\hline A22 & $4.62 \pm 0.22$ & $0.72 * *$ & $8.42 \pm 0.41$ & $0.77 * *$ & $7.31 \pm 0.06$ & $1.19 * * *$ \\
\hline A23 & $7.39 \pm 0.11$ & $1.10 * *$ & $14.76 \pm 0.96$ & $1.82 * * *$ & $2.92 \pm 0.22$ & 0.85 \\
\hline A24 & $6.27 \pm 0.21$ & $0.58 * * *$ & $26.89 \pm 0.49$ & $1.61 * * *$ & $2.35 \pm 0.34$ & 1.26 \\
\hline A25 & $2.40 \pm 0.26$ & 0.92 & $2.05 \pm 1.27$ & $0.49 *$ & $9.5 \pm 0.25$ & $1.10 * *$ \\
\hline A26 & $11.98 \pm 0.71$ & $1.12 *$ & $17.34 \pm 0.95$ & $0.99 *$ & $4.36 \pm 0.48$ & $1.68 * *$ \\
\hline A27 & $4.82 \pm 0.10$ & $0.86 * *$ & $12.29 \pm 1.29$ & $1.51^{* *}$ & $6.07 \pm 0.21$ & $1.09 *$ \\
\hline A28 & $5.67 \pm 0.31$ & 1.01 & $12.07 \pm 0.46$ & $1.57 * * *$ & $5.78 \pm 0.19$ & $1.12 *$ \\
\hline A29 & $6.30 \pm 0.20$ & 0.96 & $13.03 \pm 1.22$ & $1.35^{* *}$ & $6.05 \pm 0.02$ & $1.27 * * *$ \\
\hline
\end{tabular}

A significant difference compared the respective EAC value with TAC (* $p<0.05, * * p<0.01, * * * p<0.001)$. ${ }^{a}$ Experimental antioxidant capacity $\left(\right.$ EAC) of antioxidant mixtures expressed as Trolox equivalent $\left(\right.$ TE). Values are the mean $(n=3) \pm$ S.D. ${ }^{\text {b }}$ SE $>1$ : synergistic effect shown; SE $<1$ : no synergistic effect found.

statistically significant. The remaining three-component formulas were not greater than 1 .

Antioxidant formulas A4-A6, A11-A16 and A21-A26 contained two antioxidants. In the DPPH model. The SE values for antioxidant mixtures A6, A23 and A26 were greater than 1, shown in Fig. 2(B). A26 showed a significant synergistic effect, $\mathrm{SE}=1.12, \mathrm{EAC}=11.98 \pm 0.24 \mu \mathrm{M}$ (Table-2). In mixtures, when increasing the content of caffeic acid, the EAC value became greater than increasing other antioxidants, i.e., caffeic acid has the largest contribution to the antioxidant capacity. Pre-test indicating the reaction was essentially completed after $0.5 \mathrm{~h}$

Reducing power model: In reducing power model, all three-component formulas have shown strong synergistic effects, shown in Fig. 3(C). The experimental antioxidant capacity (EAC) of A30 was $21.16 \pm 0.16(p<0.001)$ (Table-2), which was highest EAC among three-component formulas and higher EAC than the use of any single component contained in the formula.

Two-component formulas A4, A6, A11, A15, A16, A21, A23 and A24 showed synergistic effects in the reducing power model [Fig. 3(D)]. All five combination formulas containing glutathione and caffeic acid have shown synergistic effects, A24 had the highest EAC $26.89 \pm 0.49 \mu \mathrm{M}(p<0.001)$ in reducing power model. It had experimental data to vertify that mixtures of glutathione and caffeic acid were more effective than the single antioxidants in the FRAP model ${ }^{27}$. There were five combinations contained phloretin and glutathione (A4, A11, A12, A21 and A25), among which, A4, A11 and A21 showed synergistic effects whose content of phloretin was less than $50 \%$, when the content of phloretin was higher than $50 \%$, there had no synergistic effects. Malin Hultberg ${ }^{28}$ reported the presence of flavonoid increased the total amount of glutathione in both Hela and hepatoma cell cultures and some flavonoids increased the total amount of glutathione only occured in the case of low concentrations in hepatoma cell cultures. Phloretin and caffeic acid were flavonoids, so the results were in agreement with the mechanism proposed by Malin Hultberg.

In conclusion, all formulas that exhibited synergistic effect in reducing power model contained glutathione, but when glutathione was deleted, no synergistic effect was found. It required a result that the presence of glutathione played a major role in synergistic effect in reducing power model.

Trolox equivalent antioxidant capacity model: In TEAC model, all 12 three-component formulas showed synergistic effects, shown in Fig. 4(E). Fomula A10 had the highest synergistic effect $(\mathrm{SE}=1.44, p<0.01)$.

In the two-component formulas, A4, A5, A11-A14, A21, A22 and A24-A26, showed synergistic effects, shown in Fig. 

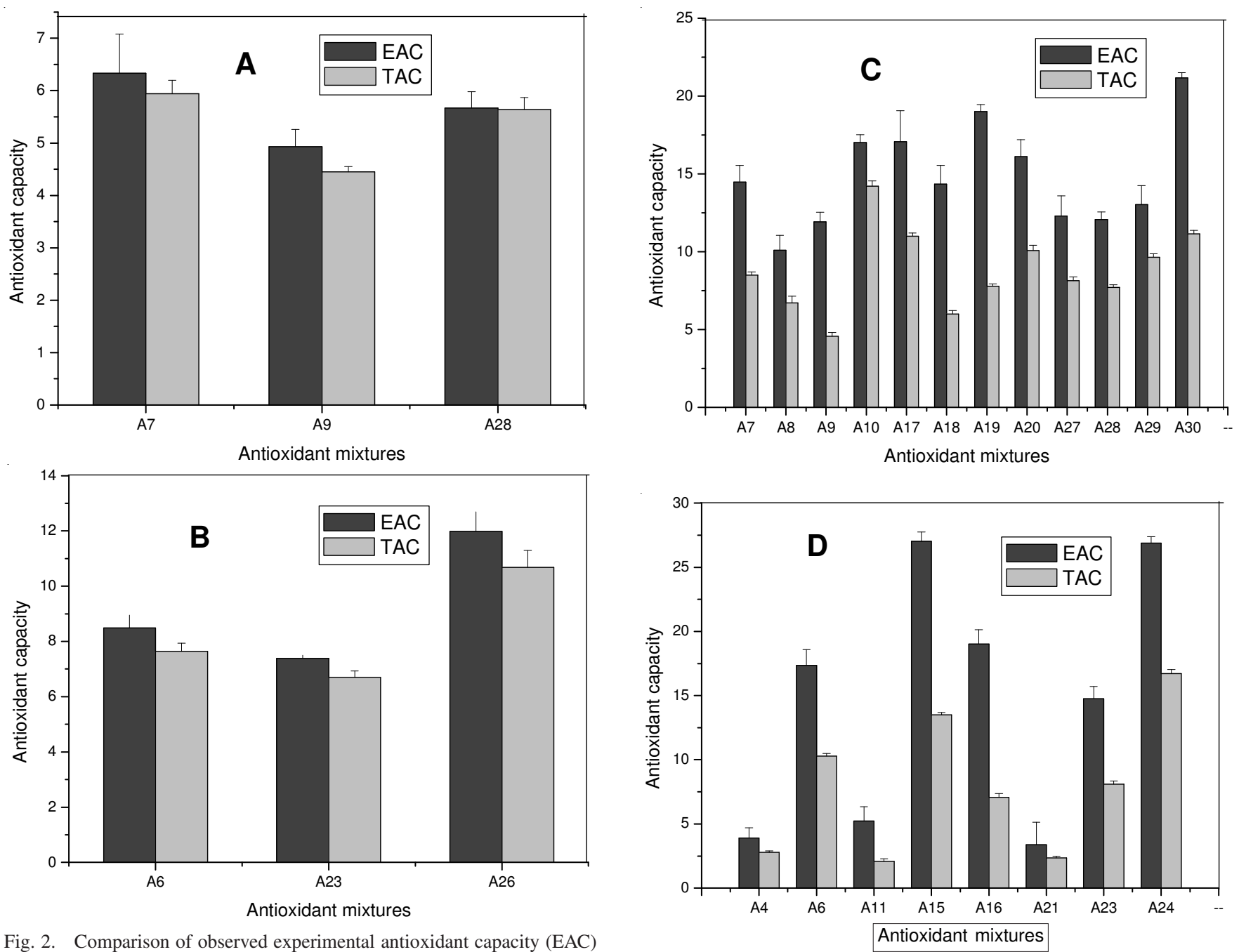

Fig. 2. Comparison of observed experimental antioxidant capacity (EAC) and theoretical antioxidant capacity (TAC) of antioxidant. (A) Threesample mixtures in DPPH model. (B) Two-sample mixtures in DPPH model. Values are means \pm SD

4(F), but A6, A15, A16 and A23 showed SE < 1, A24 had no statistically significant difference was indicated. A6, A15, A16, A23 and A24 all only contained GSH and caffeic acid and had no synergistic effects. Obviously, in TEAC model, compositions had SE when phloretin was added, i.e., phloretin occupied the main role in synergistic effect, that was similar to reducing power model.

From the experiment, it acquired that the antioxidant capacities of compound formula was great relevant to content of various components by synergistic effect analysis. Many biological actions in vivo had been attributed to antioxidant properties in vitro ${ }^{29}$. Therefore, it could not deny the significance of synergies in vitro model. The reaction mixture solution in this study here was ethanol and water, might be not conform to the reaction mixture solution was liposomes, membranes, microsomes or LDLs, making the lipohilic and hydrophilic properties of both the antioxidant and the model mechanism as important factors ${ }^{30}$. A formula in different model had different antioxidant effect and different model had different antioxidant synergism mechanism. DPPH model reflected free radical scavenging ability, the mechanism was hydrogen-donating reaction in organic phase. Caffeic acid and phloretin were polyphenols and the antioxidant efficiency of

Fig. 3. Comparison of observed experimental antioxidant capacity (EAC) and theoretical antioxidant capacity (TAC) of antioxidant. (C) Threesample mixtures in reducing power model. (D) Two-sample mixtures in reducing power model. Values are means $\pm \mathrm{SD}$

polyphenols largely depended on their hydrogen-donating ability, which was directly related to the number and reactivity of phenolic hydroxyl moieties present ${ }^{31}$. The structure-activity relationship: $o$-methoxyphenolic $>o$-hydroxyphenolic $>$ independent hydroxyl grounp from both experiments ${ }^{32}$ and theoretical calculations ${ }^{33}$. In the present work, caffeic acid which bore $o$-hydroxyphenolic is the most active one. Phloretin which bore four independent hydroxyl grounps was less active than caffeic acid, although its hydroxyl number was more than caffeic acid. Glutathione which bore sulfydryl was stronger active than hydroxyl. So the antioxidant capcacity of three antioxidants: CAF > GSH > PHL in DPPH model (Fig. 1). TEAC was used to detect total antioxidant capacity, weaker material selective compared with DPPH model and the number of active functional group was to determine the antioxidant activity of the only factor ${ }^{34}$. Phloretin bore four donating $\mathrm{H}$-atom functional groups, secondly, GSH, caffeic acid at least. The antioxidant activity order: PHL $>$ GSH $>$ CAF. In reducing power model, $\mathrm{Fe}^{3+}$ obtained the electronics provided from the antioxidants, subsequently reduced to $\mathrm{Fe}^{2+}$ in aqueous phase. In this experiment, caffeic acid and phloretin bore high electron delocalization properties of the benzene ring, but the 

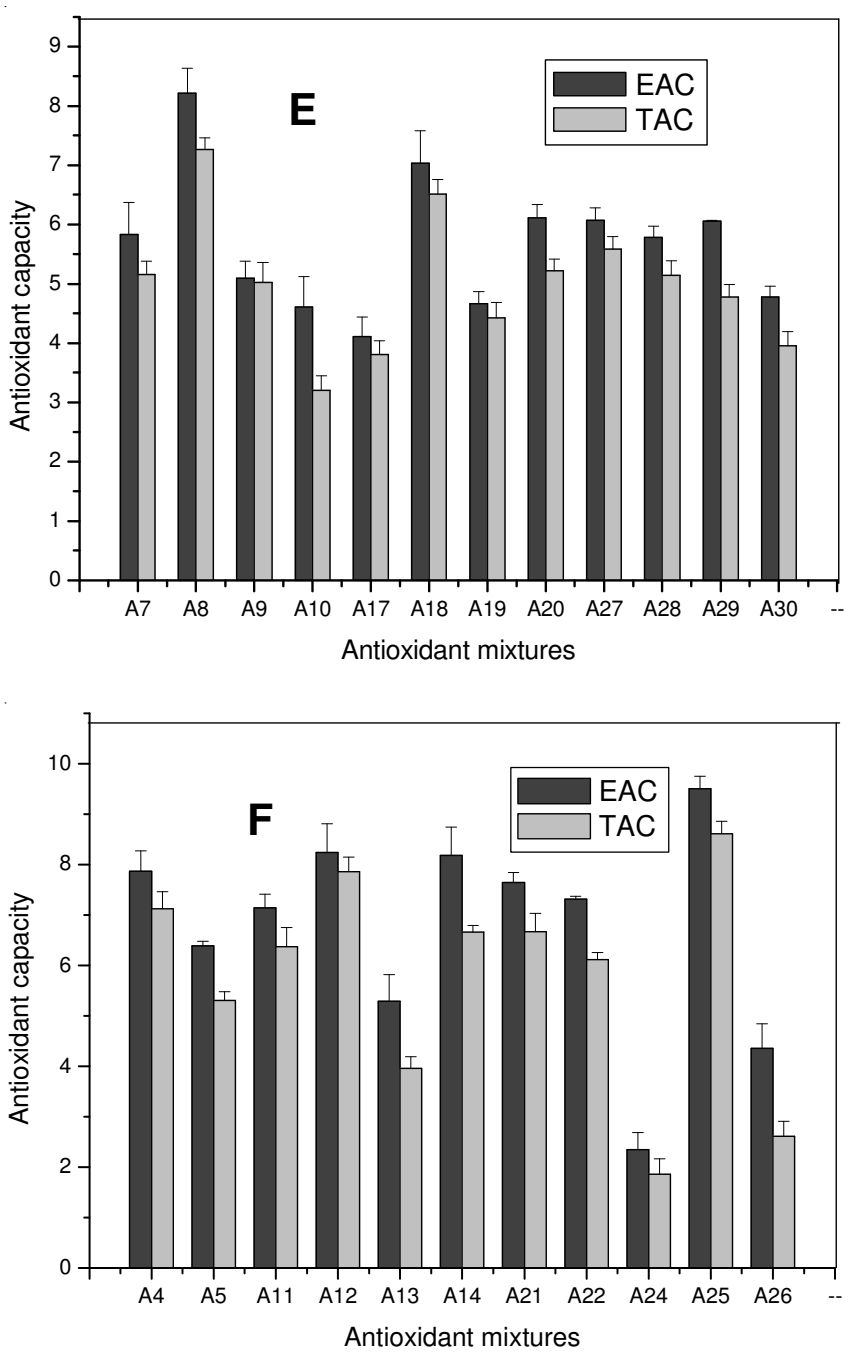

Fig. 4. Comparison of observed experimental antioxidant capacity (EAC) and theoretical antioxidant capacity (TAC) of antioxidant. (E) Threesample mixtures in TEAC model. (F) Two-sample mixtures in TEAC model. Values are means \pm SD

whole molecule of former formed a conjugated system, namely the providing electronic ability of caffeic acid was more than phloretin. GSH which bore no benzene ring was significantly less active than others. In addition, solvent polarity and the solubility of antioxidants in the solvent could influence material's antioxidant activity also and that must be considered in chemical model.

The interaction of antioxidants existed several possibilities. If an antioxidant $\mathrm{AOH}_{2}$ able of two one-electron or hydrogen-donating reactions reacted with oxidant species (OS), a free radical of the antioxidant was formed:

$$
\begin{aligned}
& \mathrm{AOH}_{2}+\mathrm{OS} \stackrel{\mathrm{H}}{\longrightarrow} \mathrm{AOH}^{\bullet}+\mathrm{HOS} \\
& \mathrm{AOH}^{\bullet}+\mathrm{OS} \stackrel{\mathrm{H}}{\longleftrightarrow} \mathrm{AO}=\mathrm{O}+\mathrm{HOS} \\
& \mathrm{AOH}_{2}+\mathrm{OS} \stackrel{\mathrm{e}}{\longleftrightarrow} \mathrm{AOH}_{2}^{+}+\mathrm{OH}^{-} \longleftrightarrow \mathrm{AOH}^{\bullet}+\mathrm{H}^{+} \\
& \mathrm{AOH}^{\bullet}+\mathrm{OS} \stackrel{\mathrm{e}}{\longleftrightarrow} \mathrm{AOH}^{+}+\mathrm{OS}^{-} \longleftrightarrow \mathrm{AO}+\mathrm{H}^{+}
\end{aligned}
$$

eqns. 1 and 2 were hydrogen-donating mechanism of single antioxidant; eqns. 3 and 4 were electron transfer mechanism of single antioxidant. If antioxidants (AO) only reacted with oxidant species (OS), respectively and no any additional reactions, until to achieve stability. There was no interaction among antioxidants, i.e., additive effects.

One of the possible reactions that all $\mathrm{AOH}^{\bullet}$ reacted with each other existed in solution, named $\mathrm{BOH}^{\bullet}$, forming a $\mathrm{AOH}-$ $\mathrm{BOH}$ adduct not reacting further with oxidant species, shown in eqn. 5 .

$$
\mathrm{AOH}^{\bullet}+\mathrm{BOH}^{\bullet} \longleftrightarrow \mathrm{AOH}-\mathrm{BOH}
$$

Antioxidant activity of the mixture of two antioxidants would be two-fold lower with respect to the situation when the two antioxidants were evaluated separately, which showed antagonistic effects.

Another possible reactions that could occur was shown in eqns. 6 and 7 and constituted one of the possible reactions of regeneration of $\mathrm{AOH}_{2}$ or $\mathrm{AOH}^{\circ}$. It could explain the synergism observed since the predominant direction of this step determined if the regeneration of the primary radical would take place. Supposing a quinone ${ }^{35}$ was final oxidized form and $\mathrm{BOH}_{2}$ was another anotioxidant coexisted in silution.

$$
\begin{aligned}
& \mathrm{AO}=\mathrm{O}+\mathrm{BOH}_{2}\left(\mathrm{BOH}^{\bullet}\right) \longleftrightarrow \mathrm{AOH}^{\bullet}+\mathrm{BOH}^{\bullet}(\mathrm{BO}=\mathrm{O})(6) \\
& \mathrm{AOH}^{\bullet}+\mathrm{BOH}_{2}\left(\mathrm{BOH}^{\bullet}\right) \longleftrightarrow \mathrm{AOH}_{2}+\mathrm{BOH}^{\bullet}(\mathrm{BO}=\mathrm{O})
\end{aligned}
$$

The regeneration of an antioxidant by another was a process that depended on several factors as the reduction potentials of the couples involved as well as their reactivities ${ }^{36}$, when the more effective compound was regenerated by the less active one eqn. 6 or eqn. 7 happened. Moreover, the extent of subadditivity would be dependent on the concentration of one of the interacting antioxidants and insensitive to change in the concentration of the other one ${ }^{37}$. Consequently, synergy was subject to content changes.

To sum up, the comprehensive effect of above factors led to the different interaction among antioxidants. Existing experiments showed that between quercetin and GSH existed reaction according to eqn. $5^{38}$ and the regeneration of Trolox occurs in vivo via GSH as cofactors ${ }^{39}$. Comprehensively considering the above factors, such as synergy law, previous studies and interaction mechanism, etc., then audaciously speculated that the antagonism might be due to eqn. 5 in this study and the synergism might be due to regeneration, to form antioxidant loop system by electrons or hydrogen-donating transfer in reducing power model and in TEAC model, see Fig. 5. But no suitable synergistic antioxidant mechanism to explain the synergy rule in DPPH model at present.

As shown in Fig. 5, in reducing power model, glutathione had the lowest antioxidant activity and rebirthed caffeic acid that had stronger antioxidant activity. When phloretin in low concentration, the primary reduction product regenerated is glutathione. The combination of $\mathrm{PHLOH}^{\circ}$ and $\mathrm{CAFOH}^{\circ}$ prevented oxidation reaction to continue and produced antagonist effect. Similarly, in TEAC model, the antioxidant activities of glutathione and caffeic acid were lower than phloretin, so phloretin was rebirthed to comply with synergy rule. The combination of $\mathrm{GSHOH}^{\bullet}$ and $\mathrm{CAFOH}^{\bullet}$ prevented oxidation reaction to continue and produced antagonist effect.

Formulation optimization: Using the integrated index evaluation method to process data and the results were used 


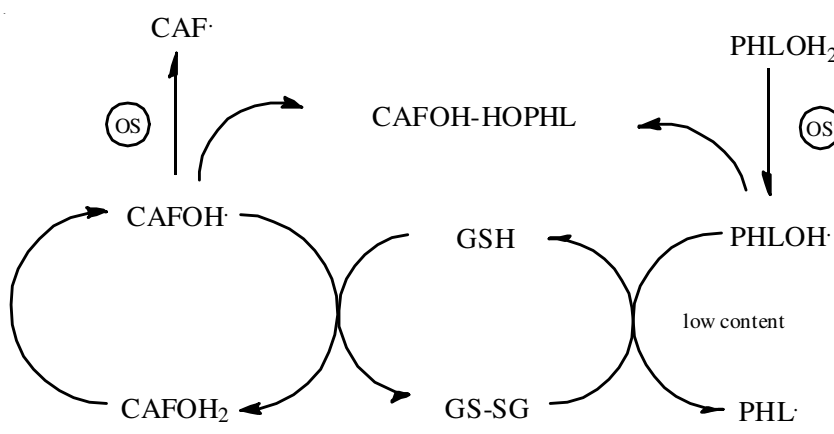

(A)

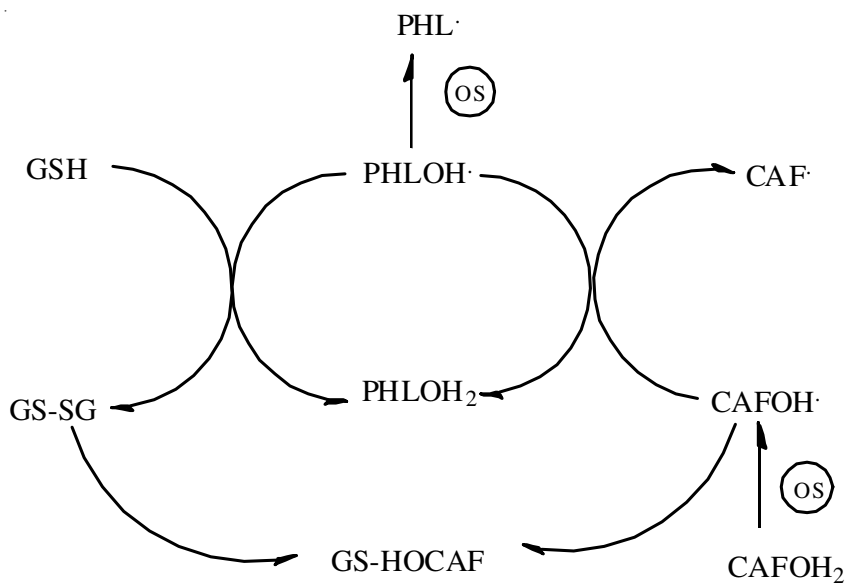

(B)

Fig. 5. (A) Synergistic antioxidant mechanism in reducing power model. (B) Synergistic antioxidant mechanism in TEAC model.PHLOH $\mathrm{PH}_{2}$ $\mathrm{CAFOH}_{2}, \mathrm{GSHOH}_{2}$ represent original antioxidants; $\mathrm{PHLOH}^{*}$, $\mathrm{CAFOH}^{\bullet}, \mathrm{GSHOH}^{\bullet}$ represent primary reduction product; $\mathrm{PHL}^{*}$, $\mathrm{CAF}^{*}, \mathrm{GS}-\mathrm{GS}$ represent secondary reduction product

as the evaluation index of the overall effect based on EAC values, contained overall desirability method (OD), principal component analysis (PCA) and full probability evaluation method (FPE). The results were shown in Table-3. It tried ODRSM, FPE-RSM and PCA-RSM method to optimize formula (Table-4)

The significance of all models was significant $(p>$ 0.0001). For FPE-RSM method, the special cubic model showed the best fitness. The "Pred R-Squared" of 0.6338 was in reasonable agreement with the "Adj R-Squared" of 0.7635. "Adeq Precision" measured the signal to noise ratio. A ratio greater than 4 was desirable. Its ratio of 17.673 indicated an adequate signal. This model could be used to navigate the design space. When PHL:GSH:CAF = 0.297:0.226:0.478, the result $\mathrm{P}_{\max }=0.039$. For PCA-RSM method, the cubic model showed the best fitness. When PHL:GSH:CAF $=0: 0: 1$, the result $\mathrm{F}_{\max }=2.894$. For OD-RSM method, the special cubic model shew the best fitness, but the "Pred R-Squared" of 0.5236 was not as close to the "Adj R-Squared" of 0.7576 as one might normally expect. This might indicate a large block effect or a possible problem with this model and/or data. When PHL:GSH:CAF $=0.299: 0.147: 0.554$, the result $\mathrm{OD}_{\max }=0.501$. Compared the results of three methods, OD method and the FPE method had a similar result. In PCA method, the weight of each model in analysis of the comprehensive principal component was given directly form the software, but the obtained weight was too objective to reflect actual weight of each model. As the limitations of the RSM itself, prediction obtained form the FPE-RSM was closer to the actual value than the prediction form OD-RSM, so it selected the result FPE-RSM. The optimal formula of phloretin $29.7 \%$, glutathione $22.6 \%$ and caffeic acid $47.8 \%$ had the hightest antioxidant activity than other formulas.

\section{Conclusion}

This work demonstrated that the mixture of phloretin, caffeic acid and glutathione possessed remarkably enhanced antioxidative efficacy and showed synergistic effect. It was also proved that this antioxidant synergism mechanism was due to the regeneration of three antioxidants. The optimal formula of phloretin $29.7 \%$, glutathione $22.6 \%$ and caffeic acid $47.8 \%$ was the most reasonable formula required from FPE-RSM method in this component system, which was examined experimentally, EAC $=7.03 \pm 0.21$ in DPPH model, $\mathrm{EAC}=19.75 \pm 0.14$ in reducing power model and $\mathrm{EAC}=$ $6.35 \pm 0.34$ in TEAC model at the middle and upper levels in this experimental design and $p=0.042$, hence providing useful information for antioxidant drug and cosmetic design. But there were still controversies about the behaviour of the mixtures and information about their possible in the interactions of more than two natural constituents was still scarce.

\section{ACKNOWLEDGEMENTS}

The authors wish to thank the free searching foundation of State Key Laboratory of Food Science and Technology (Nanchang University) in China (SKLF-TS-200914).

\section{REFERENCES}

1. L. Packer and C. Colman, New York: Wiley, pp. 14-28 (1999)

2. R.H. Liu, Am. J. Clin. Nutr., 78, 517S (2003).

3. F. Dai, W.F Chen and B. Zhou, Biochimie, 90, 1499 (2008).

4. C.S. Romano, K. Abadi, V. Repetto, A.A. Vojnov and S. Moreno, Food Chem., 115, 456 (2009).

5. E.M. Becker, G. Ntouma and L.H. Skibsted, Food Chem., 103, 1288 (2007).

6. M.T. Schroeder, E.M. Becker and L.H. Skibsted, J. Agric. Food Chem., 54, 3445 (2006).

7. D.H. Liu, J. Shi, A.C. Ibarra, Y. Kakuda and S.J. Xue, LWT-Food Sci. Technol., 41, 1344 (2008).

8. D. Darr and I. Fridovich, J. Invest. Dermatol., 102, 671 (1994).

9. M. Podda, M.G. Traber, C. Weber, L.-J. Yan and L. Packer, Free Radical Biol. Med., 24, 55 (1998).

10. H. Sies, Free Radical Biol. Med., 27, 916 (1999).

11. V. Barón and P. Muriel, Biochim. Biophys. Acta, 1472, 173 (1999).

12. N.L. Reynaert, Biochim. Biophys. Acta, 1810, 1045 (2011).

13. A. Ayer, S.X. Tan and C.M. Grant, Free Radical Biol. Med., 49, 1956 (2010).

14. B.M. Rezk, G.R.M.M Haenen, W.J.F. van der Vijgh and A. Bast, Biochem. Biophys. Res. Commun., 295, 9 (2002).

15. K. Zhang and N.P. Das, Biochem. Pharmacol., 47, 2063 (1994).

16. X.Y. Lu, Y.Y. Zeng, Y.X. Ye et al., Acta Pharm. Sin., 44, 480 (2009).

17. C.A. Rice-Evans, N.J. Miller and G. Paganga, Free Radical Biol. Med., 20, 933 (1996).

18. Y. Sato, S. Itagaki, T. Kurokawa, J. Ogura, M. Kobayashi, T. Hirano, M. Sugawara and K. Iseki, Int. J. Pharm., 403, 136 (2011).

19. K.H. Janbaz, S.A. Saeed andA.H. Gilani, Phytomedicine, 11, 424 (2004).

20. P. Molyneux, Songklanakarin J. Sci. Technol., 26, 211 (2004).

21. P. Maisuthisakul, R. Pongsawatmanit and M.H. Gordon, Food Chem., 100, 1409 (2007).

22. M. Gupta, U.K. Mazumdar and P. Gomathi, Phcog. Mag., 3, 219 (2007). 
TABLE-3

INTEGRATED INDEXES OF THREE METHODS

\begin{tabular}{|c|c|c|c|c|c|c|c|c|}
\hline \multirow{2}{*}{ Code } & \multicolumn{3}{|c|}{ Principal component } & \multicolumn{4}{|c|}{ Probit } & \multirow{2}{*}{$\mathrm{OD}^{\mathrm{c}}$} \\
\hline & (F1) & (F2) & $\left(\mathrm{F}^{\mathrm{a}}\right)$ & (P1) & (P2) & (P3) & $\left(\mathrm{P}^{\mathrm{b}}\right)$ & \\
\hline A1 & -2.193 & 0.15 & -1.755 & 0.017 & 0.013 & 0.056 & 0.035 & 0.31 \\
\hline A2 & -1.242 & 0.14 & -0.983 & 0.019 & 0.002 & 0.029 & 0.017 & 0.2 \\
\hline A3 & 3.22 & 1.287 & 2.859 & 0.081 & 0.052 & 0.007 & 0.033 & 0.39 \\
\hline A4 & -1.883 & 0.056 & -1.52 & 0.016 & 0.01 & 0.047 & 0.029 & 0.28 \\
\hline A5 & 0.081 & 0.735 & 0.203 & 0.046 & 0.031 & 0.038 & 0.036 & 0.51 \\
\hline A6 & 2.003 & 0.374 & 1.698 & 0.056 & 0.046 & 0.013 & 0.03 & 0.41 \\
\hline A7 & 0.326 & 0.282 & 0.318 & 0.041 & 0.038 & 0.035 & 0.037 & 0.5 \\
\hline A8 & -1.196 & 0.048 & -0.963 & 0.025 & 0.026 & 0.049 & 0.038 & 0.42 \\
\hline A9 & 0.039 & -0.044 & 0.023 & 0.032 & 0.031 & 0.03 & 0.031 & 0.41 \\
\hline A10 & 1.168 & 0.439 & 1.032 & 0.051 & 0.045 & 0.027 & 0.037 & 0.52 \\
\hline A11 & -1.686 & -0.299 & -1.427 & 0.012 & 0.014 & 0.043 & 0.028 & 0.25 \\
\hline A12 & -2.132 & 0.023 & -1.729 & 0.014 & 0.008 & 0.049 & 0.029 & 0.25 \\
\hline A13 & 0.14 & 0.559 & 0.218 & 0.042 & 0.027 & 0.032 & 0.031 & 0.44 \\
\hline A14 & -1.439 & 0.329 & -1.108 & 0.026 & 0.018 & 0.049 & 0.034 & 0.38 \\
\hline A15 & 1.75 & -1.986 & 1.051 & 0.021 & 0.071 & 0.013 & 0.037 & 0.33 \\
\hline A16 & 1.142 & -1.206 & 0.703 & 0.024 & 0.05 & 0.014 & 0.029 & 0.32 \\
\hline A17 & 0.893 & -0.276 & 0.674 & 0.037 & 0.045 & 0.024 & 0.034 & 0.45 \\
\hline A18 & -0.786 & -0.809 & -0.791 & 0.016 & 0.038 & 0.042 & 0.038 & 0.36 \\
\hline A19 & 0.509 & -0.987 & 0.229 & 0.024 & 0.05 & 0.028 & 0.036 & 0.41 \\
\hline A20 & -0.084 & -0.553 & -0.172 & 0.026 & 0.042 & 0.036 & 0.038 & 0.44 \\
\hline A21 & -1.915 & -0.011 & -1.559 & 0.015 & 0.009 & 0.046 & 0.028 & 0.25 \\
\hline A22 & -0.927 & 0.338 & -0.69 & 0.03 & 0.022 & 0.044 & 0.034 & 0.42 \\
\hline A23 & 1.358 & 0.297 & 1.16 & 0.048 & 0.039 & 0.017 & 0.029 & 0.42 \\
\hline A24 & 2.317 & -0.99 & 1.698 & 0.041 & 0.07 & 0.014 & 0.039 & 0.43 \\
\hline A25 & -2.502 & 0.33 & -1.973 & 0.016 & 0.005 & 0.057 & 0.032 & 0.26 \\
\hline A26 & 2.084 & 1.686 & 2.009 & 0.078 & 0.045 & 0.026 & 0.039 & 0.6 \\
\hline A27 & -0.221 & -0.006 & -0.181 & 0.032 & 0.032 & 0.036 & 0.034 & 0.44 \\
\hline A28 & 0.008 & 0.245 & 0.052 & 0.037 & 0.032 & 0.034 & 0.034 & 0.45 \\
\hline A29 & 0.138 & 0.399 & 0.187 & 0.041 & 0.034 & 0.036 & 0.036 & 0.49 \\
\hline A30 & 1.031 & -0.548 & 0.736 & 0.036 & 0.055 & 0.028 & 0.04 & 0.5 \\
\hline Total & 2.283 & 0.525 & - & - & - & - & - & - \\
\hline Variance (\%) & 76.103 & 17.504 & - & - & - & - & - & - \\
\hline Cumulative (\%) & 76.103 & 93.607 & - & - & - & - & - & - \\
\hline Weight (w) & - & - & - & 0.1 & 0.4 & 0.5 & - & - \\
\hline
\end{tabular}

\section{TABLE-4}

POLYNOMIAL EQUATION AND OPTIMIZED RESULTS OF DIFFERENT METHODS

\begin{tabular}{cccc}
\hline Way & Fitted models & \multicolumn{1}{c}{ Polynomial equation } & Optimized results \\
\hline PCA & Cubic & $\mathrm{F}=-1.82669 \mathrm{X}_{1}-1.01155 \mathrm{X}_{2}+2.89421 \mathrm{X}_{3}-0.74093 \mathrm{X}_{1} \mathrm{X}_{2}-2.94317 \mathrm{X}_{1} \mathrm{X}_{3}+$ & $\left(\mathrm{X}_{1}, \mathrm{X}_{2}, \mathrm{X}_{3}\right)=(0,0,1)$, \\
& & $0.90235 \mathrm{X}_{2} \mathrm{X}_{3}+9.13247 \mathrm{X}_{1} \mathrm{X}_{2} \mathrm{X}_{3}-0.13483 \mathrm{X}_{1} \mathrm{X}_{2}\left(\mathrm{X}_{1}-\mathrm{X}_{2}\right)-0.084638 \mathrm{X}_{1}$ & $\mathrm{~F}_{\max }=2.894$ \\
& & $\mathrm{X}_{3}\left(\mathrm{X}_{1}-\mathrm{X}_{3}\right)+6.66847 \mathrm{X}_{2} \mathrm{X}_{3}\left(\mathrm{X}_{2}-\mathrm{X}_{3}\right) ; \mathrm{R}^{2}=0.9729$ & \\
FPE & Special cubic & $\mathrm{P}=+0.034110 \mathrm{X}_{1}+0.017598 \mathrm{X}_{2}+0.036016 \mathrm{X}_{3}+0.014179 \mathrm{X}_{1} \mathrm{X}_{2}-$ & $\left(\mathrm{X}_{1}, \mathrm{X}_{2}, \mathrm{X}_{3}\right)=(0.297,0.226,0.478)$, \\
& & $2.41693 \mathrm{E}-003 \mathrm{X}_{1} \mathrm{X}_{3}+0.022604 \mathrm{X}_{2} \mathrm{X}_{3}+0.10385 \mathrm{X}_{1} \mathrm{X}_{2} \mathrm{X}_{3} ; \mathrm{R}^{2}=0.8125$ & $\mathrm{P}_{\max }=0.039$ \\
OD & Special cubic & $\mathrm{OD}=+0.27559 \mathrm{X}_{1}+0.22865 \mathrm{X}_{2}+0.43997 \mathrm{X}_{3}+0.029416 \mathrm{X}_{1} \mathrm{X}_{2}+0.44188$ & $\left(\mathrm{X}_{1}, \mathrm{X}_{2}, \mathrm{X}_{3}\right)=(0.299,0.147,0.554)$, \\
& & $\mathrm{X}_{1} \mathrm{X}_{3}+0.20386 \mathrm{X}_{2} \mathrm{X}_{3}+2.07252 \mathrm{X}_{1} \mathrm{X}_{2} \mathrm{X}_{3} ; \mathrm{R}^{2}=0.8078$ & $\mathrm{OD}_{\max }=0.501$ \\
\hline
\end{tabular}

23. R. Berg van den, G.R.M.M. Haenen, H. Berg van den, W. Vijgh vander and A. Bast, Food Chem., 70, 391 (2000).

24. A. Zulueta, M.J. Esteve and A. Frígola, Food Chem., 114, 310 (2009).

25. M.J.T.J. Arts, J.S. Dallinga, H.P. Voss, G.R.M.M. Haenen and A. Bast, Food Chem., 88, 567 (2004).

26. B. Fuhrman, N. Volkova, M. Rosenblat and M. Aviram, Antioxid. Redox Signaling, 2, 491 (2000).

27. C.D. Capitani, A.C.L. Carvalho, P.B. Botelho, M.M. Carrapeiro and I.A. Castro, Eur. J. Lipid Sci. Technol., 111, 1100 (2009).

28. M. Hultberg and B. Hultberg, Chem. Biol. Interact., 163, 192 (2006).

29. A. Luximon-Ramma, T. Bahorun, M. Soobratee and O.I. Aruoma, J. Agric. Food Chem., 50, 5042 (2002).

30. E. Niki, N. Noguchi, H. Tsuchihashi and N. Gotoh, Am. J. Chin. Nutr., 62, 1322 (1995).

31. B. Turan, A. Gulsen, D.P. Makris and P. Kefalas, Food Res. Int., 40, 819 (2007).
32. M. Foti and G. Ruberto, J. Agric. Food Chem., 49, 342 (2001).

33. J.S. Wright, E.R. Johnson and G.A. DiLabio, J. Am. Chem. Soc., 123, 1173 (2001).

34. F. Nanjo, M. Mori, K. Goto and Y. Hara, Biosci. Biotechnol. Biochem., 63, 1621 (1999).

35. A.W. Boots, X. Boots, G.R. Haenen and A. Bast, Biochim. Biophys. Res. Commun., 308, 560 (2003).

36. G.R. Buettner, Arch. Biochem. Biophys., 300, 535 (1993).

37. A. Blauz, T. Pilaszek, A. Grzelak, A. Dragan and G. Bartosz, Food Chem. Toxicol., 46, 2365 (2008).

38. A.W. Boots, J.M. Balk, A. Bast and G.R. Haenen, Biochim. Biophys. Res. Commun., 338, 923 (2005).

39. R.C. Rose and A.M. Bode, FASEB J., 7, 1135 (1993). 